\title{
LOS FACTORES TERAPÉUTICOS: ¿QUÉ ES LO QUE PRODUCE CAMBIOS EN LOS GRUPOS?
}

\section{JOSÉ FRANCISCO CAMPOS VIDAL}

TEU del Área de Trabajo Social y Servicios Sociales, Vicedecano de Trabajo Social de la Facultad de Filosofía y Letras. Universidad de las Islas Baleares.

\section{LOS FACTORES TERAPÉUTICOS: ¿QUÉ ES LO QUE PRO- DUCE CAMBIOS EN LOS GRUPOS?}

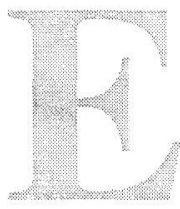

Entendemos al Trabajo Social de Grupo como aquella rama del Trabajo Social que se caracteriza por el uso del grupo como medio y contexto para la modificación de las circunstancias personales generadoras de malestar o circunstancias que impiden a los componentes del grupo desarroIlar sus capacidades, afectos, relaciones o responsabilidades sociales. Así mismo, el Trabajo Social de grupo se caracteriza por el uso del grupo como medio e instrumento para la modificación del entorno y contexto social, operando en el marco de colectivos o territorio singulares y diferenciados.

Así pues, reconocemos e identificamos tres dimensiones en las aguas territoriales del Trabajo Social de Grupo:

a) una dimensión individual en la cual el Trabajo Social de Grupo se centra en la singular problemática de cada uno de los sujetos que componen el grupo. Se trata de los grupos centrados sobre sí mismos, creados ad hoc por el propio Trabajo Social, con objetivos de cambio individual y que han dado origen al modelo terapéutico de Vinter (1969) o al modelo socioterapéutico/socioeducativo a los cuales hace referencia Rossell (1997).

b) Una dimensión grupal, en la cual el objeto de atención es el grupo en sí mismo, el grupo como un sistema social en situación de dificultad. Puede tratarse del grupo de autoayuda o el grupo de acción social, en ambos casos, grupos preexistentes en un territorio y en un colectivo concreto. Esta dimensión estimuló la creación del modelo recíproco de Schwartz (1971).

c) Y, por último, una dimensión comunitaria en la cual el grupo existe, opera y persigue objetivos para cambiar, modificar o mejorar 
situaciones que afectan a otros grupos, a otros colectivos o a territorios singulares, y que ha sido el impulsor del modelo de objetivos sociales o el modelo de asesoramiento comunitario de Egan (1981).

Entendemos que esta triple vertiente está asumida en la definición, ya clásica, que hace Konopka' acerca de lo que es el Trabajo Social de Grupo: "un método de Trabajo Social que ayuda a los individuos, por medio de experiencias intencionadas en grupo, a mejorar su funcionamiento social y a enfrentarse de una manera más efectiva con sus problemas personales, de grupo o de comunidad".

Desde la perspectiva de los grupos centrados sobre el cliente, nos interesa interrogarnos sobre las siguientes cuestiones: ¿cómo ayuda el Trabajo Social de Grupo a sus clientes? ¿Cómo se mejora el funcionamiento social? ¿De qué manera es posible que los individuos se enfrenten con más eficacia a sus problemas? Expresándolo de otra forma, ¿se dan condiciones necesarias fundamentales que posibilitan el desarrollo de procesos de cambio? En el caso de que así sea, ¿cuáles son esas condiciones, bajo qué parámetros se expresan y cuál es su naturaleza?

Generalmente, la bibliografía de referencia, ${ }^{2}$ en lengua castellana e italiana en relación al Trabajo Social de Grupo, ha prestado escasa atención al desarrollo de los fenómenos intra-grupales relacionados con el cambio. En general, los trabajos que se encuentran a nuestra disposición abundan en consideraciones teórico-metodológicas ligadas a la teoría de grupo, generalmente bajo el epígrafe general de "Dinámica de Grupo" (Shaw, 1980): estructura, redes de comunicación, roles, liderazgo, conflicto grupal y ciclo evolutivo del grupo.

El conjunto de las investigaciones desarrolladas en este sentido, asentadas en los laboratorios de grupo bajo el formato general de "T-Group" o grupos de encuentro y crecimiento, nada nos dicen de la dinámica del cambio en la medida de que el cambio no ha sido su objeto concreto de investigación. Desde la perspectiva del Trabajo Social, es evidente que se hace necesario el conocimiento del sujeto objeto de observación -en nuestro caso, el grupo-, aunque, en este caso, el sujeto observado pre-

Konopka, G. (1969): Trabajo Social de Grupo. Euramérica, Madrid.

2 Prestamos atención especial a los trabajos de Contreras (1980), Vinter (1967), Kisnerman (1973), Konopka (1969, 1973), Kisnerman y Mustieles (1997), Williamson (1961, 1969) o Di Carlo, et. alt. (1962, 1997), Brown (1988), Tschorne (1990, 1993), Baten (1969), Domenech (1998) y Ruiz (1996), en lengua castellana, y a los trabajos de Zini y Modini (1999), Bocolan, Carbonaro y Bennici (1990) y Heap (1986), en lengua italiana. 
senta una particularidad que incrementa su nivel de complejidad: es un sujeto que quiere cambiar o que necesita cambiar.

Desde una perspectiva profesional, el grupo de Trabajo Social tiene y adquiere un sentido en la medida que, como tal, se orienta hacia un cambio: identifica problemas o situaciones problemáticas ${ }^{3}$, determina su voluntad de cambio, formula objetivos de cambio, determina tareas encaminadas a alcanzar el cambio y evalúa los cambios resultantes. A nuestro juicio, los grupos de Trabajo Social son algo más que un laboratorio de dinámica grupal, independientemente de que los fenómenos intra-grupales aparezcan y se desarrollen en toda su extensión. Así pues, desde nuestro punto de vista, se hace dramáticamente necesario determinar cuáles son los fenómenos que favorecen el cambio o, si se prefiere, las condiciones necesarias que deben desplegarse para que el cambio sea posible.

Queremos en este momento hacer algunas consideraciones con el objeto de clarificar la intención de este trabajo. Cuando hablamos de condiciones necesarias o fenómenos que favorecen el cambio, no estamos haciendo referencia a técnicas orientadas al cambio. A nuestro entender, una técnica, siendo un procedimiento concreto que ha demostrado su efectividad para conseguir un fin concreto, podrá impulsar o inhibir el desarrollo de los fenómenos grupales de cambio. A nuestro juicio, existen factores de cambio o, si se prefiere, "factores terapéuticos" que, siendo condición necesaria, aunque no suficiente, están potencialmente presentes en todo proceso de cambio en el marco del Trabajo Social de Grupo. Así pues, afirmamos que la técnica en sí misma no cambia nada, no "cura" nada, sino que más bien cataliza cambios que afectan a mecanismos cognitivos, emocionales, relacionales o conductuales, permitiendo a los sujetos experimentar experiencias internas y relacionales que permitirán cambios relacionales e internos. $\mathrm{Si}$

3 Es evidente que cualquier situación-problema, sea cual sea su origen y naturaleza, impacta en mayor o menor medida sobre los subsistemas constitutivos del sujeto (CASIC: conductual, afectivo, somático, interpersonal y cognoscitivo) y sobre la red social en el cual éste se desarrolla. Entendemos por necesidad a aquella situación problema caracte. rizada por la precariedad de los medios de subsistencia y supervivencia del sujeto o sistema implicado; entendemos por dificultad a aquella situación problema en la cual es posible una solución satisfactoria pero que, en la cual, el sujeto o el sistema implicado no dispone o no está en condiciones de activar sus recursos y capacidades personales, sistémicos o medioambientales; entendemos por problema a aquella situación que no tendrá solución y que, manteniéndose indefinidamente en el tiempo, va a generar numerosas necesidades, dificultades o conflictos; entendemos por conflicto a aquella situación problema en la cual sujetos y sistemas se encuentran enfrentados y que, toda solución significa, en primera instancia, la resolución de un dilema por alguna de las partes. 
desconocemos qué es lo que favorece el cambio, nunca podremos determinar con precisión la idoneidad de la estructura, el contexto y el proceso grupal, y tampoco la congruencia de la aplicación técnica con los objetivos personales y grupales.

Por otra parte, cabe considerar que los fenómenos relacionales que acontecen en el grupo y que favorecen o inhiben el cambio poseen una naturaleza propia e independiente del marco teórico de observación desde el cual el Trabajador Social ${ }^{4}$ lea, interprete y opere con el grupo. Ello significa que el profesional, independientemente de su marco referencial teórico (psicodinámico, cognitivista, enfoque conductual clásico, psicodramático, rogersiano, transaccional, realista o racional-emotivo, como ejemplo de los marcos fundamentales), no puede ignorar la existencia de una pluralidad de factores terapéuticos y que, algunos de ellos, pueden no ser consistentemente considerados en el desarrollo del modelo terapéutico $0^{5}$ Y, si nos lo permiten, queremos señalar un último elemento que incrementa el nivel de complejidad. Los factores terapéuticos que, a continuación señalaremos, están íntimamente condicionados por dos elementos: la vida evolutiva del grupo y el contexto de intervención en el cual el grupo opera. Desde el punto de vista de la vida evolutiva del grupo, los factores terapéuticos presentan una lógica secuencial particular ligada a los distintos estadios del mismo: la cohesión grupal no actúa como factor de cambio en los primeros estadios y sí en los intermedios y finales, mientras que la universalidad o el mantenimiento de la esperanza son factores precoces ya en los primeros estadios de formación del grupo. El contexto de intervención (asistencial, in-formativo, asesoramiento, clínico, control y evaluación) favorecerá especialmente el desarrollo de aquellos factores terapéuticos más acordes con la naturaleza del propio contexto: relacionales, emocionales, conductuales o cognitivos. Así, por ejemplo, un contexto clínico enfatizará factores terapéuticos de naturaleza emocional, cognitiva y relacional, mientras que un contexto in-formativo muy probablemente destacará factores terapéuticos de naturaleza conductual, cognitiva e interaccional.

Bien, veamos de qué hablamos cuando hacemos referencia a los "factores terapéuticos". El abanico de factores terapéuticos está recogido de forma fragmentada en los trabajos de Weiner-Davis (1989), Watzlawick, Weakland y Fisch (1982), de Shazer (1999), Selekman (1996) y Neuburger (1997), Madanes (1997) y, con especial atención en relación al trabajo de grupo, en las obras de Yalom y Vinogradov

Entiéndase Trabajador y Trabajadora Social, indistintamente.

Por ejemplo, un enfoque conductual ortodoxo no considerará la expresión de emociones o el mantenimiento y el fomento de la esperanza, como elementos a considerar y fomentar. 
(1996), Yalom(2000), Corey (1996), Bloch y Crouch(1985) y Stockton y Hulse (1980), Gutiérrez et. al. (1997), Aznar et. al. (1995), y Tevan (1998), entre otros. A nuestro modesto juicio, los trabajos de Yalom y Vinogradov, Bloch y Crouch, Stockton y Hulse, y, en cierta medida, Corey sintetizan y resumen con claridad la naturaleza de los factores terapéuticos.

Intentado responder a la pregunta ¿qué sucede en el seno de un grupo que hace que los clientes cambien?, nos centraremos en los fenómenos relacionales y psíquicos que se desencadenan en los grupos, desde una perspectiva individual y colectiva. Evidentemente, el desarrollo de actividades grupales facilitará o inhibirá tales fenómenos; sin embargo, en esta ocasión, no trataremos la idoneidad o no de las tareas que se desarrollan en el grupo, sino más bien en los factores que permiten producir el cambio individual y grupal.

\section{LOS FACTORES TERAPÉUTICOS}

\subsection{Infundir y mantener la esperanza}

El infundir y mantener la esperanza es crucial en todo Trabajo Social de grupo. Yalom (1996:25 y ss.; 2000:26) señala que la esperanza no sólo se requiere para mantener al cliente en el grupo, para que puedan tener efecto otros factores terapéuticos, sino en que la fe en un modo de tratamiento puede por sí misma ser efectiva terapéuticamente. De un modo subyacente, plantea el problema de la predeterminación o la capacidad de decisión y la responsabilidad de las personas. Una perspectiva que mantenga la génesis predeterminada de la naturaleza humana difícilmente se apoyará sobre la base de la esperanza. Una perspectiva que se sostenga sobre la tesis de que las personas tienen capacidad de decisión sobre su futuro, de que las personas son responsables de sus actos y que, por tanto, tienen la capacidad de cambiar, influirá enormemente sobre la idea de la esperanza, dicho de otro modo, sobre la firme creencia de que el cambio es posible ${ }^{6}$.

Las investigaciones ${ }^{7}$ de los terapeutas encuadrados en el modelo centrado en la solución, de orientación sistémica, han demostrado que una elevada expectativa de ayuda antes del proceso de tratamiento se correlaciona significativamente con un resultado positivo del mismo.

" Madanes, C. (1997): "Una terapia de acción social", en Violencia Masculina. Granica, Buenos Aires, pp. 17-27.

7 Kim Berg. I.; Miller, S. (1996): Trabajando con el problema del alcohol. Gedisa, Barcelona. 
Hay que considerar también la masiva documentación sobre la eficacia de la creencia en el cambio a partir de la construcción de narraciones alternativas elaboradas conjuntamente entre los clientes y los profesionales ${ }^{8}$.

Es evidente que el mantenimiento y el estímulo de la esperanza, el desarrollo de la idea de que el cambio es posible, es un factor terapéutico inicial que en sí mismo es extremadamente frágil en los comienzos de la relación intra-grupal. Por ello, los Trabajadores Sociales deberemos mantener y transmitir mensajes claros y reiterados que sustenten la esperanza y la idea de que es posible hacer algo, de que es posible cambiar. Se trata de introducir o reforzar en el sistema de creencias de los miembros del grupo tanto la idea de cambio con la creencia de que el cambio es posible a través del trabajo de grupo y, consecuentemente, de la relación interpersonal en el marco del mismo. Este mensaje y la creencia que lo sostiene debe plantearse tanto en los estadios de preparación pre-grupal como en los distintos momentos de la negociación del contrato a los cuales hace referencia Brown (1988:58 y ss.).

Por otra parte, cabe señalar que los grupos de Trabajo Social, especialmente los grupos socioterapéuticos y socioeducativos a los cuales hace referencia Rossell ${ }^{9}$, posibilitan el re-agrupamiento de personas que, compartiendo situaciones de dificultad equivalentes en relación a sus características, se sitúan en momentos distintos, circunstancias particulares distintas, narraciones distintas e intentos de solución -exitosos o excepcionales- también distintos. La constelación de singularidades relacionadas con las situaciones problemáticas y las personas que las experimentan posibilita un caleidoscopio de situaciones que son susceptibles de ejemplificar y demostrar que nada es permanente y que el cambio es posible. Por ejemplo, una pareja de jóvenes padres con un hijo afectado de diabetes, pueden observar y compartir con otra joven pareja en idéntica situación que ya lleva un año de experiencia en el manejo de la situación problema. Esta particularidad es intrínseca al nivel de intervención grupal, dado que la posibilidad de compartir entre iguales -padres jóvenes, por ejemplo- no se da en el nivel individualfamiliar.

Yalom (2000:27) señala que el estímulo y el mantenimiento de la esperanza es una de las variables estructurales de los grupos de autoayuda: narraciones de recaídas y recuperaciones permiten visualizar

3 Neimeyer, R. y Mahoney, M. (1998): Constructivismo en Psicoterapia. Paidós, Barcelona.

9 Rossell, T. (1997): "Trabajo Social de Grupo: grupos socioeducativos y grupos socioterapéuticos", en Cuadernos de Trabajo Social n ${ }^{\circ} 11$, Universidad Complutense de Madrid, Madrid, pp. 103-122. 
a los miembros noveles del grupo de autoayuda que lo que uno está viviendo no es permanente y que el cambio es posible.

Hasta el momento hemos hecho referencia al factor del estímulo y el mantenimiento de la esperanza, fundamentalmente desde la perspectiva de los partícipes del grupo. Sería un error considerar exclusivamente que este factor es atribuible a una de las partes. En términos sistémicos, un grupo de Trabajo Social es definible como un sistema de ayuda y como tal está constituido por el subsistema clientes y el subsistema Trabajador Social.

Los Trabajadores Sociales de grupo no sólo deben tener muy en cuenta este potente mensaje "el cambio es posible", también deben creerlo firmemente, ya que sin esperanza no hay posibilidades de cambio.

Esta cuestión, la creencia de que el cambio es posible, apunta a un elemento crítico del contenido de la relación terapéutica que desarrollan los Trabajadores Sociales de grupo: la congruencia o la discrepancia entre los mensajes digitales (verbales) que el profesional lanza al grupo y que afirman que es posible el cambio, y los mensajes analógicos (no verbales) que acompañan a los primeros. En primer lugar, queremos destacar que si un Trabajador Social no cree firmemente que el cambio es posible, independientemente de que intente transmitir este mensaje a sus clientes o que despliegue todo un arsenal técnico orientado a provocar cambios, analógicamente emitirá un mensaje de "no cambio", congruente con su creencia íntima. Esta contradicción comunicacional, ligada teóricamente a la Teoría del Doble Vínculo, planteada por Bateson y colaboradore ${ }^{10}$, inevitable e inconscientemente será trasmitida a los miembros del grupo. En segundo lugar, e insistiendo en esta tesis, si un Trabajador Social no cree que el cambio es posible, puede llegar a provocar un intercambio comunicacional del tipo "profecía de autocumplimiento": no creo que tú puedas cambiar $\$$ no me empeño en acompañar y facilitar el cambio $\ddagger$ el otro -cliente- no experimenta los cambios "esperados" y no cambia $\ddagger$ yo confirmo que tú no puedes cambiar. La trampa de esta secuencia comunicacionalrelacional se centra en el hecho de que es el propio profesional el que provoca, sin ser consciente de ello, la conducta del cliente, es decir, el que mantiene al cliente en la situación de "no cambio".

10 Bateson, G.; Jackson, D.; Haley, H.; Weakland, J.: "Hacia una teoría de la esquizofrenia", en Behavioral Science, vol.1, número 4, octubre de 1956. Existe una traducción en Iengua castellana en: VV.AA. $(1971,1974)$ Interacción familiar. Ed. Tiempo Contemporáneo. Buenos Aires. 


\subsection{Universalidad}

El Trabajo Social ha reconocido históricamente la singularidad de la vivencia que experimenta el cliente, la particularidad del significado y del impacto que provoca sobre el cliente y su sistema de referencia la situación vivida (Biestek, 1966). Esta singularidad, este significado particular experimentado por el cliente, desde una perspectiva emocional, cognitiva, relacional y conductual, es generalmente hiperdimensionada por el propio cliente. La propia autosingularización siempre tiene una referencia real, independientemente del significado particular para cada uno o de la constelación de fantasías, autopercepciones, pensamientos o impulsos que el sujeto experimenta: lo que le está sucediento, sólo le sucede a él. Siendo esta última afirmación correcta, es falso deducir que la autopercepción de esta singularidad es excepcional y única. Desgraciadamente, la mayoría de clientes que están atravesando situaciones de crisis creen que lo que les está sucediendo "sólo les sucede a ellos" y consecuentemente, "nadie puede hacerse cargo o comprender lo que están viviendo": dolor, ideas y fantasías inaceptables, conductas inapropiadas, autodestructivas o negligentes, etc.

Las investigaciones desarrolladas por Caplan (1994, 1997) y Slaikeu (1988) dejan patente una característica universal que se manifiesta en las personas que atraviesan momentos de crisis: su tendencia al aislamiento social y, consecuentemente, la pérdida significativa de sus redes de apoyo social. Este fenómeno impide a los sujetos experimentar cualquier tipo de aprendizaje interpersonal que proporcione mecanismos o estrategias de corrección de la hiperdimensionada unicidad a la cual hemos hecho referencia anteriormente, por lo que es frecuente que se desencadene un proceso de aislamiento que se retroalimenta a sí mismo: cuanto más solo me siento, más me aíslo + cuanto más me aíslo, más solo me siento.

Las primeras etapas de la experiencia grupal proporcionan una experiencia externa y relacional que posibilita un cambio interno, un cambio en la percepción de sí mismo y una re-colocación de esa hiperdimensionada unicidad. La relación en el marco del grupo, identificando personas en circunstancias similares -mismo malestar, mismo dolor, mismas dificultades y misma voluntad de cambio-, posibilita romper el aislamiento, romper la singularidad, es decir, reconocerse a sí mismo en el otro y reconocer al otro y en el otro un vínculo de unidad.

Este reconocimiento recíproco es generalmente experimentado como un fuerte alivio, es decir, la aceptación de que "no estoy solo, hay más como yo, más que me entienden". Sin duda, se constituye en un significativo corrector de la ansiedad. 
Estas experiencias internas revierten en el sujeto y en el propio grupo en la medida en que naturalizan la situación vivida por cada sujeto, le proporcionan una percepción de mayor normalidad, le permiten frenar los procesos degradadores de la auto-imagen, estimulan la auto-aceptación, la ayuda mutua, refuerzan el vínculo grupal y refuerzan el mensaje de que el cambio es posible.

Yalom (2000:30) señala que la universalidad necesariamente se combina con el resto de factores terapéuticos en la medida que es un facilitador, un catalizador en la aparición y desarrollo de los demás. Nosotros entendemos que la universalidad es el primer factor terapéutico que facilita la cohesión grupal. Sin duda, una cohesión frágil e incipiente en los primeros estadios del grupo, pero que se constituye en el denominador común a partir del cual es posible la aceptación de los otros, la exploración y la exposición de narraciones y experiencias traumáticas, la ayuda mutua y la aceptación de feedbacks correctores.

\subsection{Transmisión de información}

Por transmisión de información entendemos a todos aquellos procesos grupales didácticos que permiten a los miembros del grupo comprender la naturaleza de su situación de dificultad, sus características, su curso evolutivo previsible y los cambios o el impacto que esa situación puede generar sobre el sistema de referencia del sujeto (familia y red relacional primaria). Así mismo, la transmisión de información considera en su seno las instrucciones o el asesoramiento en relación a cómo debe obrar el cliente ante una determinada situación o circunstancia relacionada con su situación problemática. Los contextos de intervención in-formativos utilizan de forma preponderante la transmisión de información, aunque ésta no es exclusiva de dicho contexto.

El impacto de este factor sobre los miembros del grupo no sólo se centra en su componente cognitivo, es decir, el saber más en relación a lo que sucede, a su evolución y a su significado, sino que también permite la alteración de conductas y pensamientos destructivos (como, por ejemplo, la clarificación en relación a temores infundados sobre el desarrollo de enfermedades en pacientes de $\mathrm{VIH}^{11}$ ), preparar a las personas para el afrontamiento de situaciones futuras (como, por ejemplo, la preparación del duelo en grupos de familiares con enfermos terminales) o la comprensión y el manejo de las características de la situación problemática (como, por ejemplo, la revelación sobre la adopción que los padres deberán efectuar a sus hijos) o la comprensión de la 
transitoriedad del dolor que se está experimentado, el cual desembocará en un período posterior de calma (como, por ejemplo, los grupos de padres con hijos con discapacidad visual). ${ }^{12}$

En todos estos elementos, generalmente la educación y la formación están implícitas; de hecho, numerosas experiencias demuestran que los Trabajadores Sociales han hecho de la instrucción formal una herramienta de frecuente utilización, especialmente en contextos informativos. La literatura reciente sobre trabajo de grupo abunda en las descripciones de grupos especializados para clientes que padecen algún desorden específico, o que afrontan alguna crisis vital, como por ejemplo la anorexia ${ }^{13}$, toxicodependencias ${ }^{14}$, diabete ${ }^{15}$, cuidados intensivos $^{16}$, adopción y acogimiento ${ }^{17}$ o la inserción social entre otros.

Desde nuestro punto de vista, la transmisión de información es un potente factor terapéutico en la medida en que incide directa y arrolladoramente sobre el "no saber". Desconocer qué sucederá o qué es lo que está sucediendo impide a los sujetos abastecerse de un marco cognitivo-temporal de seguridad establecido sobre la condición de previsibilidad. De hecho, el no saber ha sido siempre la fuente de la búsqueda del conocimiento y la explicación de los fenómenos que nos acontecen y que se desarrollan en nuestro medio ambiente: ha sido la condición para el nacimiento del conocimiento y la explicación del mundo en sus diversas manifestaciones: científicas, religiosas, etc.

El "no saber", la incertidumbre, es una fuente inagotable de desestabilización del equilibrio personal y sistémico, así como una fuente generadora de ansiedad. Frieda Fromm-Reichman subraya el papel de la incertidumbre en la producción de ansiedad ${ }^{18}$. Señala que el darse cuenta de que uno no es el director de escena de su propia obra, que las propias percepciones y la conducta están controladas por fuerzas que ni

12 Cardona, J. (1998): Programa de asesoramiento psicosocial para padres de niños con discapacidad visual. ONCE, Delegación Territorial de Baleares. Palma, Mimeo.

13 Aznar, J. et alt. (1995): "Un año de grupo de autoayuda con familiares de enfermos mentales en Zaragoza". Revista Trabajo Social y Salud $n^{\circ} 20$.

14 Selekman, M. (1996): "El grupo de padres centrado en la solución", en Abrir caminos para el cambio, Gedisa, Barcelona.

15 Gutiérrez et alt. (1997): "Educación grupal con diabéticos de Motril. Experiencias desde el Trabajo Social sanitario". Revista Trabajo Social y salud n" 26.

10 Tevan, R. (1998): "Grupos de padres de una unidad de cuidados intensivos". Revista Trabajo Social y Salud $n^{\circ} 30$.

17 Triseliotis, J. (1994): El Trabajo de Grupo en la adopción y acogimiento familiar. Ministerio de Asuntos Sociales, Madrid.

18 Fromm-Riechman, F. (1950): Principles of intensive Psychoterapy, citado en Yalom, $\mathrm{I}$. Ob. Cit. pp.35. 
entiende ni comprende, es en sí mismo una fuente importante de ansiedad. Significa, en definitiva, el reconocimiento de la pérdida del control sobre la propia vida, independientemente de que el control de la vida de uno sea, evidentemente, relativo. Esto es lo que sucede con los clientes psiquiátricos que se encuentra en período de recuperación de una crisis aguda: su tristeza y los síntomas depresivos que manifiestan están íntimamente relacionados con el miedo y la ansiedad resultante de la incertidumbre respecto a la fuente y el significado de sus síntomas. Este fenómeno fue descrito con maestría en la obra literaria de Malcom Lowry, Bajo el volcán (Tusquets Ed., 1997): “...El horror de una realidad insoportable, esto no debe ser muy distinto, se dijo, del sufrimiento de un demente en aquellos momentos en que, sentado con mansedumbre en los patios del manicomio, la locura deja de ser su refugio...".

Así pues, el "saber" lo que está sucediendo, lo que va a suceder y el cómo sucede permiten al sujeto partícipe del grupo recuperar la percepción del control de los acontecimientos y del autocontrol. Si uno cree o percibe que mantiene un cierto control respecto a lo que sucede, entonces está en mejores condiciones para ejercer la capacidad de decir y, si uno puede decidir, puede orientar esta capacidad de decisión hacia el cambio asertivo, evitando en este caso que los intentos de solución orientados al cambio se conviertan, como expresan los autores de la Escuela de Palo Alto, en un problema, o si se prefiere, en "el problema".

\subsection{Altruismo}

"Una persona muere y se le da oportunidad de visitar el Cielo y el Infiemo. Al entrar en el Infierno, ve un espectáculo muy extraño: hay comida por doquier y, sin embargo, los residentes parecen famélicos. Dirige sus pasos adonde está el Diablo, y le pregunta: ¿Por qué se mueren todos de hambre, habiendo tanta comida por todas partes?

El Diablo responde: La gente no tiene codos. No podemos comer.

La persona visita luego el Cielo y se encuentra con una situación similar: abunda la comida y la gente carece de codos. Pero existe una notable diferencia: está bien alimentada. El visitante pregunta a un Angel: ¿Por qué en el Infierno la gente no tiene codos y se muere de hambre, pero aquí, en el Cielo, aunque la situación es la misma, la gente se ve robusta y bien alimentada?. 
El Angel lo mira directamente a los ojos y contesta: Nos damos de comer unos a otros.

\author{
Charles Fishman, 1994 \\ Terapia estructural Intensiva
}

Tratamiento de familias en su contexto social.

Amorrortu, pp. 279-280

Con anterioridad, hemos hecho referencia al grupo de Trabajo Social como un sistema de ayuda constituido por dos subsistemas claramente diferenciados: el subsistema clientes y el subsistema Trabajador Social. La participación de ambos en el sistema de ayuda adquiere un sentido y una intencionalidad que deberá clarificarse lo más prontamente posible a través de la definición del contexto de intervención. Sin embargo, a priori, los roles que desarrollan ambos subsistemas en relación a sus individualidades vienen definidos por dos perfiles definidos en el metacontexto que permite o facilita el encuentro (nos referimos al servicio o la constelación de servicios implicados, así como a la red relación de referencia de cada uno de los subsistemas implicados). En primer lugar, el rol del cliente es un rol auto-asumido, caracterizado por "la persona que necesita de algo" -sea material o inmaterial-. Este rol auto-asumido (y, en parte, atribuido) se sustenta en la creencia que se apoya en la falta de capacidad, es decir, se trata de personas "que no pueden dar", en todo caso, sólo pueden recibir.

El contra-rol, en este caso, atribuido al Trabajador Social (y, en parte, asumido) está caracterizado por el de la "persona que posee algo" -sea material o inmaterial-, cuya creencia está sustentada sobre la idea de competencia. Se trata, en definitiva, de la o las "personas que dan".

Como ya hemos expuesto anteriormente, los clientes en crisis, en tanto que acentúan su tendencia al aislamiento y a empobrecer sus vínculos de apoyo social, no tienen oportunidades de experimentar situaciones relacionales en las cuales ese rol, de "persona que necesita de algo" y "persona que no puede dar nada", sea puesto en cuestión. Se trata de un cliente con tendencia al aislamiento o claramente aislado.

El cliente que participa en un grupo de Trabajo Social es envuelto en un escenario -el escenario grupal- en el cual el aislamiento no es posible. En dicho escenario no puede dejar de comportarse ni, en congruencia, dejar de comunicarse. Se trata por tanto, de un escenario comunicacional en el cual el sujeto deja de estar aislado.

La conducta altruista significa el desarrollo de un proceso, puntual o permanente, meditado o impulsivo, de dar al otro aquello que uno cree 
que el otro necesita en un momento determinado, sin una finalidad estratégica, sin mediar contraprestación, compensación o ganancia. Sin embargo, en el seno de los grupos centrados sobre el cliente, cuando uno da algo (información, apoyo, afecto, ayuda material, feedback, etc.), está a su vez recibiendo también algo.

William Glasser, creador del modelo de la terapia de la realidad, es un psiquiatra entrenado analíticamente que se sintió insatisfecho con la psiquiatría convencional. La terapia de la realidad enseña al cliente cómo satisfacer dos necesidades psicológicas básicas: 1) la necesidad de amar y de ser amado, y 2) la necesidad de sentirse útil o de mantener un nivel satisfactorio de conducta (Glasser, 1965). Más tarde, fundió las dos en una única necesidad básica: la necesidad de identidad (Glasser,1979).

Para Glasser, la identidad del sujeto juega un aspecto crucial. Un individuo debe hallar cierta identidad, aun cuando ésta sea negativa o de fracaso. La satisfacción de las dos necesidades básicas contribuye a una identidad positiva o de éxito. Hay momentos en la vida en los que el mundo y las situaciones cambian, lo que exige que las personas aprendan una y otra vez a satisfacer sus necesidades bajo diferentes condiciones y tensiones. Los individuos deben vincularse a otras personas si quieren afrontar eficazmente sus necesidades. Si esta unión se interrumpe en algún momento de la vida, no tarda en experimentarse la incapacidad de satisfacer las necesidades básicas. Glasser concluía "que todas las personas que tienen alguna clase de problema psiquiátrico grave carecen de una vinculación satisfactoria con otra persona, lo que las incapacita para satisfacer sus necesidades".

Su modelo destaca los comportamientos sociales como un elemento muy importante para la buena salud mental. Glasser (1979:31-32) cree que "debemos sentirnos vinculados a otras personas, como mínimo a una, pero con la esperanza de llegar a relacionarnos con muchas más. En todos los momentos de nuestra vida debemos tener al menos una persona que se preocupe de nosotros y a la que, por nuestra parte, prestemos toda nuestra atención. Si no contamos con ella, no podremos satisfacer nuestras necesidades básicas». Para poder lograr este objetivo, los clientes deben sentirse vinculados a otras personas a las que aman y por las que son amados. Deben mantener asimismo un nivel satisfactorio de conducta que les permita sentirse valiosos para sí mismos y para otros. Si no satisfacen estas necesidades básicas - que suelen ir unidas en su frustración o en su satisfacción-, los clientes sentirán aflicción y malestar y, como consecuencia, tratarán de aplicar métodos no realistas para remediar sus males.

Veamos con más detalle estas dos necesidades básicas: "La primera es la necesidad de amar y ser amado. En todas sus formas, que se 
ordenan desde la amistad, el amor maternal, el amor familiar, al amor conyugal, es una necesidad que nos induce a la actividad constante en busca de satisfacción. Desde que nacemos hasta la ancianidad necesitamos amar y ser amados, y durante la existencia entera la salud y la felicidad dependerán de la capacidad de conseguirlo. Amar nosotros solamente, o ser amados sin reciprocidad no es bastante; son precisas ambas condiciones. Cuando no podemos satisfacer la necesidad total de amar, forzosamente sufriremos, reaccionando con varios síntomas, desde el ligero desasosiego, ansiedad y depresión, hasta la completa retirada del mundo que nos rodea" (Glasser, 1979:35).

De la misma importancia -sigue Glasser-es la necesidad de sentirnos útiles y de que los demás juzguen que lo somos. "(...) Que se nos ame o no, para ser dignos debemos mantener un tipo de comportamiento satisfactorio. Para lograrlo tenemos que aprender a corregirnos cuando obramos mal y a estimarnos cuando actuamos bien. Si no evaluamos el propio comportamiento, o luego de haberlo evaluado no hacemos por mejorar la conducta en los puntos en que se encuentra por debajo de las normas que nos hemos fijado, no satisfaremos la necesidad de ser dignos y habremos de sufrir tan agudamente como cuando no amamos o no somos amados. Las costumbres, las normas, los valores o el comportamiento bueno o malo están estrechamente relacionados con la satisfacción de la necesidad de estima de uno mismo"(Glasser, 1979:36).

Así pues, en el marco de la relación grupal, la conducta altruista posibilita la satisfacción de la necesidad de sentirse útil y la vinculación a los demás, o como señalan los teóricos de la comunicación humana ${ }^{19}$, satisfacer la necesidad de confirmación. De hecho, la experiencia relacional altruista rompe la autopercepción de sí mismo como sujeto necesitado, no capacitado para dar algo y de sujeto sin valor. El compartir problemas similares en un grupo posibilita desarrollar un contexto relacional en el cual se dan numerosas experiencias y situaciones que estimulan y posibilitan la ayuda mutua y la conducta altruista. El altruismo es un modelo de conducta venerable en la mayoría de sistemas sociales: la gente necesita sentir que es necesaria y útil. Desde la perspectiva de la práctica del Trabajo Social, es extremadamente difícil que una relación profesional en un nivel individualizado permita al cliente sentirse útil; dicho en otras palabras, la relación diática Trabajador Social/Cliente no es precisamente la más idónea como para que el cliente "ayude" al Trabajador Social20.

19) Watzlawik, P.; Helmick, J.; Jackson, D.(1983): Teoria de la comunicación humana. Herder, pp. 80 y ss.

20. Los obsequios y detalles que numerosos clientes realizan al finalizar una relación profesional, no sólo siguen la lógica de una muestra simbólica de agradecimiento, sino que 
El impacto recíproco de la conducta altruista, en tanto que factor terapéutico de gran potencia, es múltiple. Para el que recibe: apoyo emocional, aceptación, confirmación, información, ayuda en las estrategias de cambio, feedbacks correctores, comprensión. El significado de estos elementos posee un valor añadido en la medida de que son conductas altruistas emitidas por un igual, por una persona real, en el mundo real que está atravesando una situación similar. Para el que da, la conducta altruista se constituye en una experiencia relacional y externa que permite un cambio interno: la conducta altruista desarrolla una configuración de la auto-imagen a través de una auto-percepción que se apoya en la capacidad y que apunta hacia una revalorización significativa de la autoestima.

\subsection{Recapitulación correctiva de isomorfismos}

Es frecuente que los grupos de Trabajo Social se constituyan con clientes que atesoran historias familiares altamente insatisfactorias o que sus problemas, necesidades, dificultades o conflictos sólo adquieran significación diagnóstica a partir de la comprensión de la estructura familiar de origen.

En el marco de la relación interpersonal grupal, y pasados los primeros estadios de la vida evolutiva del mismo, los partícipes del grupo tenderán a mostrarse tal y como son, con sus capacidades y sus dificultades. En algunos grupos, podemos observar una correlación significativa entre la naturaleza del problema y las particulares configuraciones de los grupos familiares de pertenencia (recuérdese que un cliente entra a formar parte de un grupo centrado sobre el cliente a través de una derivación, es decir, tras un trabajo de evaluación diagnóstica). Dicho en otros términos, uno tiene la tendencia a mostrarse tal cual es, y ese "ser" está mediatizado por lo aprendido e incorporado en el marco relacional en el cual uno, entendido en términos relacionales, ha construido su identidad y ha aprendido las pautas de relación y conducta social-cultural.

$\mathrm{Si}$, como ya sabemos, un grupo es en sí mismo un microcosmos social, éste posee reglas de funcionamiento y relación que mantienen analogías con el sistema familiar de referencia de cada uno de los miembros del mismo. Yalom y Vinogradov (1996:30) señalan que existen roles y situaciones semejantes a las que existen y se desarrollan en las familias: figuras parentales de autoridad, iguales, intensas relaciones emocionales, conflictos, disputas, reclamos de confirmación, etc. 
Obviamente, ocurren fenómenos similares en trabajo individual y familiar, pero el grupo proporciona un número enormemente mayor y toda una variedad de posibilidades de recapitulación.

El concepto de isomorfismo es un concepto esencial para los Trabajadores Sociales que trabajan en contextos contemporáneos, es decir, en el aquí y el ahora. Isomorfismo significa, simplemente, similitud de forma. Dos o más estructuras complejas pueden ser trazadas en superposición, de manera que cada parte de una estructura tenga una parte correspondiente de la otra, una parte que desempeñe un papel similar en ésa. El concepto de isomorfismo ayuda al profesional a percibir las similitudes estructurales entre las pautas fundamentales en los diferentes contextos en que opera una persona. Desde esta perspectiva puede observarse que una pauta de conducta en el interior de la familia se reproduce con otros en el seno del grupo. Según Fishman, ${ }^{21}$ el concepto de isomorfismo es especialmente útil y potente en la medida en que, cuanto más disfuncional sea un sistema familiar, tanto más posibilidad habrá de que isomorfos invadan otros contextos. Lo importante en el trabajo del grupo será la recapitulación correctiva, el cambio de las pautas rígidas, impenetrables y congeladas en los sistemas familiares. En este caso, el profesional debe examinar y cuestionar los roles fijos existentes, así como las pautas transaccionales repetitivas entre el sujeto y el grupo, induciendo y alentado nuevos comportamientos.

La tesis del cambio a través de la modificación de las pautas isomormas ya aparece en Erickson, el cual sostenía que los pequeños cambios desencadenaban otros mayores (Gordon y Meyers-Anderson, 1981). Una vez que se estimula a los clientes a valorar los cambios mínimos, es probable que realicen otros cambios: las partes de un sistema familiar están interconectadas de tal manera que un pequeño cambio en una parte del sistema repercute y produce modificaciones en las otras partes.

En un estudio realizado para el National Institute on Drug Abuse, Szapocznik y sus colegas ${ }^{22}$ (Szapocznik, Kurtines, Foote, Pérez-Vidal y Hervis) proporcionaron una base empírica a la idea de que los pequeños cambios pueden conducir a cambios mayores en el sistema familiar.

Los investigadores utilizaron dos grupos de sujetos. Uno de ellos estaba compuesto solamente por los adolescentes drogadictos, y el otro por adolescentes drogadictos y sus familias. Ambos grupos recibieron

21 Fishman, H.C. (1994): Terapia estructural intensiva. Tratamiento de familias en su contexto social. Amorrortu. Buenos Aires, pp. 34-39.

32 Sclekman, A. (1996): Abriendo caminos para el cambio. Gedisa, Barcelona,pp.41-42. 
un tratamiento de terapia estratégica breve de familia. Szapocznik y sus colegas descubrieron que el grupo compuesto por un solo miembro cumplió igualmente bien todos los pasos del tratamiento hasta finalizar los tres años de seguimiento. Dos importantes hallazgos surgieron de este estudio: 1) es posible cambiar el sistema familiar entero a través de uno sólo de sus miembros, y 2) no es necesario incorporar al tratamiento a todos los miembros de la familia para cambiar al cliente.

Este último hallazgo refuta el viejo precepto del enfoque sistémico según el cual todos los familiares que viven bajo el mismo techo deben participar en el tratamiento si se desea producir cambios en el cliente.

\subsection{Socialización}

El aprendizaje social es un factor terapéutico que opera en todos los grupos centrados sobre el cliente, independientemente del tipo de habilidades desplegadas o la naturaleza de los cambios planificados o deseados. La interacción grupal que se sustenta sobre continuas espirales de retroalimentación comunicacional, facilita y posibilita el continuo ensayo y error de nuevas conductas, conductas diferentes, nuevas habilidades y modelos de relación distintos. El despliegue y la exhibición de conductas, ideas, valores y pautas de comportamientos, sean éstas apropiadas o inapropiadas, recibirá a su vez numerosos feedbacks correctores o confirmadores que no son ni neutrales ni inocuos al resto de partícipes del grupo. Ello permite conocer y evaluar el impacto de las propuestas relacionales o de las conductas propias que uno desarrolla. Si uno es incapaz de mantener un contacto visual o de aceptar un cumplido, más pronto o más tarde recibirá información precisa en relación a esta dificultad. Estos "espejos" en los cuales el miembro del grupo se ve reflejado facilitan la oportunidad del cambio, es decir, permiten al sujeto ensayar nuevas o diferentes formas de conducta, relación o cognición, más apropiadas, más satisfactorias o más asertivas.

\subsection{Conducta imitativa}

La inmersión en un grupo proporciona a los clientes la observación de conductas apropiadas e inapropiadas, ideas, sentimientos y emociones que impactan a nivel individual y colectivo, modelos de relación y comunicación diversos de los cuales aprender e incorporar o que sirven de ejemplo para no incorporarlo en la constelación cognitiva y comportamental de uno mismo.

Es evidente, y así lo recoge la mayoría de la literatura profesional, que el Trabajador Social conductor de un grupo de Trabajo Social, se 
constituye en primera instancia en un poderoso modelo al cual observar o imitar. De hecho, Corey (1995:93-16) desarrolla un amplio repertorio de estilos de liderazgo, advirtiendo que la naturaleza del estilo propio del profesional influirá de forma decisiva en la relación interpersonal y, consecuentemente, en la consecución de las metas individuales y grupales. A nuestro juicio, ésta sigue siendo una variable -aunque no la únicadeterminante en el marco de la relación de ayuda grupal. El estilo, la imagen corporal, el clima emocional y la conducta del profesional favorecerán o inhibirán el desarrollo de los factores terapéuticos.

Sin embargo, los clientes en el seno del grupo no mantienen una relación unidireccional respecto al profesional, más bien todo lo contrario: la esencia del grupo es la relación multipolar. Si eso es así, cabe esperar que los miembros del grupo ejerzan su influencia unos sobre otros y que, en consecuencia, tengan la posibilidad de observar, ensayar y aprender o rechazar formas distintas de comprender lo que está sucediendo, de afrontar problemas, de expresar emociones, de entender la vida, de expresarse, de razonar, de relacionarse y de comportarse.

\subsection{Expresión de emociones}

El componente emocional inherente y derivado de toda situación de dificultad no es, a estas alturas del conocimiento, discutido por ninguna escuela de pensamiento, independientemente de que alguna de ellas, como es el caso del conductismo, no lo considere como un objeto de observación (en todo caso lo será la emoción expresada -medible-, pero no la emoción individuada).

La atención prestada por algunos enfoques, en mayor o menor medida, como el Psicodrama, la Gestalt, el Análisis Transaccional o la Terapia Racional Emotiva, han llevado, a nuestro juicio, a numerosos profesionales a interpretar que el objetivo de las intervenciones grupales se centraba principalmente en el desbloqueo de los sentimientos y las emociones ligadas a acontecimientos traumáticos, las cuales impiden a las personas alcanzar cambios satisfactorios. A nuestro juicio ésta es una interpretación errónea.

La expresión de emociones en el marco del grupo es un factor terapéutico que, ligado a la universalidad y la cohesión grupal, genera un significativo alivio, una descarga emocional que es reconfortante, pero que no genera necesariamente cambio alguno. Llorar, enfadarse, emocionarse, entristecerse, siendo un expresión de autenticidad y una revelación de la intimidad emocional, es un factor necesario aunque no suficiente para el cambio. 
Aunque la investigación sobre la valoración del cliente de los factores terapéuticos revela la importancia de la expresión de sentimientos, la investigación también sugiere requisitos importantes. En un estudio con doscientos diez participantes en grupos de encuentro, Yalom ${ }^{23} \mathrm{y}$ colaboradores encontraron que la expresión de sentimientos era necesaria para un buen resultado, pero no era suficiente. Los miembros que se referían a la importancia exclusiva de la expresión de sentimientos, de hecho, eran, con mayor probabilidad, los que habían tenido una experiencia negativa en el grupo. Aquellos que habían tenido una experiencia de crecimiento personal normalmente la asociaban con alguna forma de aprendizaje cognitivo. Las conclusiones demostraban que la aireación de los problemas, por uno mismo o de uno mismo, no fue considerada por los clientes como muy útil. La expresión de sentimientos efectiva fue vinculada a otros factores. En primer lugar, era parte de un proceso interpersonal: los miembros del grupo expresaban sus emociones, lo hacían en un contexto social. En segundo lugar, se demostraba que la expresión de sentimientos era factible en la medida de que el grupo estaba cohesionado.

A nuestro juicio la expresión de emociones apunta a tres núcleos básicos. En primer lugar, expresar emociones y afecto es esencial para el mantenimiento de la vida afectiva grupal. Independientemente de los objetivos que un grupo se marque, el desarrollo del mismo está ligado al equilibrio inestable entre dos factores: la tarea, es decir, aquellas actividades que permiten al grupo y a sus miembros alcanzar los objetivos y metas propuestas, y la vida afectiva grupal, es decir, aquellos componentes relacionales y emocionales que permiten al grupo relacionarse y existir como tal y en los cuales la tarea pasa a un segundo plano (aspiraciones personales, reconocimiento, apoyo, control, conflicto, etc.). Sin un clima relacional favorable, es imposible alcanzar las metas grupales $y$, a su vez, las metas grupales no pueden alcanzarse sin un clima emocional favorable.

En segundo lugar, la expresión de emociones permite a los miembros del grupo compartir con los demás el mundo interior. Ser aceptado incondicionalmente posibilita romper el estigma o la etiqueta que cada uno soporta y ser aceptado en tanto que ser humano singular, que sufre, se entristece, se alegra o se irrita.

En tercer lugar, considerar que en numerosas ocasiones el proceso de cambio personal exige y requiere revivir una situación dolorosa $\mathrm{o}$, si nos permiten el atrevimiento, requiere de la inducción a la crisis con tal de romper los equilibrios homeostáticos hiperactivados que imposibili-

23. Yalom, I. (2000): Psicoterapia existencial y Terapia de Grupo. Paidós Barcelona, pp. 42-43. 
tan el cambio. En ambos casos, la expresión de los sentimientos y emociones no puede ser considerada más que una expresión saludable, una reacción congruente y saludable con la situación vivida.

\subsection{Cohesión}

La cohesión ha sido investigada especialmente desde la perspectiva de la estructura y dinámica grupales. Corey (1995:148), citando a Yalom, señala que las investigaciones en el campo de la clínica grupal demuestran que la cohesión es un fuerte determinante de los resultados terapéuticos del grupo. Si la universalidad es el primer factor cohesivo en los estadios iniciales del grupo, la cohesión, entendida en el sentido más abarcativo del término, implicará el conjunto de fuerzas resultantes que hace posible que el grupo sea atractivo para sus miembros. Dado que no deseamos hacer una descripción de la cohesión como fenómeno estructural del grupo, ${ }^{24}$ vamos sólo a destacarla en tanto que factor terapéutico.

En primer lugar, cabe señalar que la cohesión posibilita la construcción de una meta-individualidad, una identidad colectiva, un "nosotros grupal" que posee una valencia incluyente en relación a sus partícipes y una valencia excluyente en relación a terceros. Desde esta perspectiva, la cohesión estimula y favorece relación de inclusión y filiación, es el soporte del mito grupal y da una imagen imaginaria acerca de lo que el grupo es para sus miembros. Filiación y pertenencia son factores intersubjetivos que estimulan la lealtad grupal (so pena de la exclusión), el compromiso entre los miembros, la complicidad tanto en Ias relaciones como en el desarrollo de tareas y la responsabilidad individual y colectiva en relación al grupo mismo.

En segundo lugar, señalar que los factores mencionados son el acicate para el desarrollo de relaciones de ayuda mutua, colaboración, apoyo, solución de problemas y afrontamiento de conflictos intra-grupales. $\mathrm{Y}$, en tercer lugar, la cohesión actúa como una fuerza unificadora y protectora. Sólo tras su experimentación subjetiva, las personas que forman el grupo están en condiciones de arriesgar y compartir las circunstancias, pensamientos, acciones y conductas dolorosas, vergonzosas o inapropiadas.

Corey (1995:149) señala que la cohesión no es el resultado del azar o de la pura relación interpersonal, sino más bien el compromiso del profesional y los miembros del grupo para dar los pasos necesarios que conducen al sentimiento de unidad grupal. Sin la percepción de este

24 Para tal menester, ver Shaw, M.(1980): Dinámica de Grupo. Herder, Barcelona. 
"nosotros grupal" las personas se retraen, no arriesgan, se defienden de las autoimágenes distorsionadas y se mueven en la superficialidad de sus situaciones problema. Sin cohesión no hay auto-apertura ni seguridad de aceptación incondicional. Como señala Yalom, la cohesión es la condición necesaria para el desarrollo de los demás factores terapéuticos y el desarrollo de un trabajo efectivo.

\subsection{Aprendizaje interpersonal}

Desde la perspectiva del modelo sistémico, el grupo es conceptualizado como un sistema (González, 1995:221 246). El grupo es una organización interdependiente en la que la conducta y la expresión de cada uno influye y es influida por el otro. La interacción en el grupo consiste en un acción de mutua influencia, una interacción que tiene lugar entre los integrantes de un grupo.

Cuando un grupo de personas se encuentra, comienza entre ellas una interacción que se plasma mediante la comunicación, que incluye mensajes verbales y no verbales, en los niveles de contenido y relación, a través de los cuales una persona emite un mensaje que genera una reacción en el destinatario, reacción que se transforma, a su vez, en un mensaje, que influye sobre el primero, y así sucesivamente (o en palabras de Bateson, cada mensaje es, a la vez, estímulo, respuesta y refuerzo).

La confirmación de la autodefinición es, probablemente, el factor que pesa más en el desarrollo y la estabilidad mental, de los que hasta ahora se han detectado en el estudio de la comunicación. Sin este efecto confirmador no se habría desarrollado la comunicación humana más que lo imprescindible para los intercambios de protección y supervivencia, no habría motivos para comunicarse por la comunicación misma. Gran parte de nuestras comunicaciones tienen un propósito confirmador. En este punto coinciden las tesis sistémicas y cognitivas.

Parece que, a parte de mero intercambio de información, el ser humano tiene que comunicarse con los otros a fin de verificar su autopercepción. La evidencia experimental de este supuesto es grande (deprivación sensorial y estabilidad emocional, por ejemplo).

La definición que cada miembro del grupo hace de sí mismo puede tener, por parte del otro, tres respuestas: confirmación, rechazo y desconfirmación, por medio de las cuales, los otros responden al primero cómo le ven, y éste cómo ve al otro y cómo ve que el otro le ve a él, etc., mensajes que a su vez pueden ser confirmados, rechazados o desconfirmados. Estos mensajes se desarrollan en base a lo que R. Laing denominó "Niveles de Percepción Interpersonal" (Laing, 1988:13-32). 
El concepto de "Niveles de Percepción" hace referencia a que mi experiencia la desarrollo no sólo en base a "la visión que yo tengo de mí mismo" (ego), o a "la visión que tengo del otro", sino a "la visión de la visión que el otro tiene de mî", es decir, "cómo yo creo que el otro me ve". A este tercer nivel lo llamaremos "metaperspectiva". En realidad, no puedo verme como los otros me ven, pero constantemente supongo que ellos me ven de determinadas maneras, y constantemente actúo a la luz de las actitudes, opiniones, necesidades, etc., reales o supuestas, que el otro tiene respecto a mí. Estas distintas formas de actuar de cara al otro son alteraciones de identidad, y en la medida en que me convierto "en otro para ti", dichas alteraciones de mi identidad son re-interiorizadas por mí para transformarse en multifacéticas metaidentidades ("ese otro que supongo que soy para el otro").

Así pues, la visión que el otro tiene de mí es tan importante, al menos en las relaciones personales estrechas, como la visión que yo tengo de mí mismo. En el mejor de los casos, ambas visiones son más o menos similares.

La conducta del otro hacia mí, para que pase a formar parte de mi experiencia personal, la debo percibir e interpretar de acuerdo con cierto conjunto de criterios personales. El ser humano aprende a estructurar sus percepciones dentro de la familia, como un subsistema que interactúa con la cultura de su contexto, las instituciones conexas y la cultura global.

En el marco del grupo, también se generan procesos de aprendizaje interpersonal que influyen significativamente en la identidad del sujeto y en la satisfacción de sus necesidades psicosociales. Yalom señala que: "Esto no quiere decir que todos los clientes, ni siquiera la mayoría que entran en el trabajo de grupo, busquen explícitamente ayuda en sus relaciones interpersonales. Sin embargo, el desplazamiento de la meta, desde el alivio del sufrimiento al cambio en el funcionamiento interpersonal es un primer paso esencial en el proceso terapéutico dinámico. No se trata exclusivamente de aliviar el síntoma o el malestar, sino de pensar o hacer de una forma diferente, con y en relación a los demás. “ (Yalom, 2000:54).

Todo ello implica que, en el marco del trabajo grupal, se proporciona el contexto dinámico para que cada miembro del grupo manifieste sus formas de entender el mundo y de relacionarse con él. Así, la relación interpersonal, el desarrollo de actividades, la manifestación de dificultades, la exposición y el riesgo de la intimidad y los intentos de solución de problemas no sólo impactan al sujeto mismo sino también al grupo.

De esta manera, mediatizada por los niveles de percepción inter- 
personal, el sujeto aprende de sí mismo, de los otros y de las relaciones entre y con los otros. Este fenómeno terapéutico se escapa al control del profesional, aunque la habilidad para identificar y plantear el cómo aprovechar terapéuticamente el comportamiento interpersonal, visto en el microcosmos social de un grupo pequeño, es una de las principales tareas de un programa de formación para Trabajadores Sociales de Grupo. ${ }^{25}$ La clave para comprender el significado del aprendizaje interpersonal radica, a nuestro juicio, en el hecho de que es el grupo en su conjunto el que se hace responsable del proceso de solución de problemas (tareas) y del mantenimiento de la vida afectiva del mismo: unos aprenden de otros, unos influyen a otros, y viceversa.

\section{CONCLUSIÓN}

Como habrá podido observarse, los denominados "factores terapéuticos" abarcan los aspectos relacionales, comunicacionales, cognitivos, conductuales y emocionales, de origen e implicación intra e interpersonales, que se despliegan en el desarrollo de un Trabajo Social de Grupo eficaz. Cabría ahora señalar, aunque no lo haremos, en qué momento del ciclo evolutivo grupal dichos factores emergen o son especialmente significativos y qué implicaciones se derivan de la relación entre dichos factores y la estructura del grupo y su dinámica. Así mismo, se hace necesario establecer una correlación entre cada uno de los factores terapéuticos y los procedimientos y técnicas de intervención grupal, determinando la congruencia entre la emergencia del factor terapéutico y la/s técnica/s específica/s. Sin duda, estos dos últimos problemas son objeto de uno o varios trabajos, más extensos y, probablemente, más detallados.

Queremos finalizar enlazando con las preguntas que nos planteábamos al principio. No es posible desarrollar un Trabajo Social de Grupo eficaz exclusivamente a través de la aplicación de técnicas estandarizadas, y tampoco es posible desarrollar un trabajo orientado hacia el cambio con la simple comprensión intelectual de lo que está sucediendo en el seno del grupo. Un Trabajo Social eficaz requiere, entre otros elementos, del conocimiento y la comprensión de los mecanismos terapéuticos, de las técnicas que estimulan estos factores terapéuticos y, especialmente, del hacerse cargo del impacto emocional, conductual, cognitivo e interpersonal que dichos factores terapéuticos provocan en las personas integrantes del grupo y en el grupo como un todo.

25 Permitame que lo diga bien claro: con la aplicación de las lamentables "Directrices Generales Propias conducentes a la obtención del Título de Diplomado en Trabajo Social", mucho me temo que se trata de un objetivo imposible de alcanzar. 


\section{BIBLIOGRAFÍA}

AZNAR, J. et alt. (1995): "Un año de grupo de autoayuda con familiares de enfermos mentales en Zaragoza”. Revista Trabajo Social y Salud n ${ }^{\circ} 20$.

BIESTEK, F. (1966): Las relaciones del casework. Aguilar, Madrid.

BLOCH, S.; CROUCH, E. (1985): Therapeutic Factors in Group Psycotherapy. Oxford University Press, Oxford.

BROWN, A. (1988): Treball de Grup. Pòrtic, Barcelona.

CAPLAN, G.; CAPLAN, R. (1997): Consulta y colaboración en salud mental. Paidós, Barcelona.

CAPLAN, G.(1994): Aspectos preventivos en salud mental. Paidós, Barcelona.

CARDONA, J. (1998): Programa de asesoramiento psicosocial para padres de niños con discapacidad visual. ONCE, Delegación Territorial de Baleares. Palma, Mimeo.

COREY, G. (1996): Teoría y práctica de la Terapia de Grupo. Desclée de Brouwer, Bilbao.

DE SHAZER, S. (1999): En un origen las palabras eran magia. Gedisa, Barcelona.

EGAN,G. (1981): El orientador experto. Wasdword International Iberoamérica, México.

FISHMAN, H.C. (1994): Terapia estructural intensiva. Tratamiento de fanilias en su contexto social. Amorrortu, Buenos Aires.

GLASSER, W. (1979): Reality Therapy: un nuevo camino para la psiquiatría. Narcea, Madrid.

GONZÁLEZ, P.(1995): Orientaciones teóricas fundamentales en psicología de los Grupos. EUB, Barcelona.

GORDON, D.; MEYERS-ANDERSON, M. (1981): Phoenix: Therapeutics Patterns of Milton Erikson. Meta. Cupertino, California.

GUTIÉRREZ et alt. (1997): “Educación grupal con diabéticos de Motril. Experiencias desde el Trabajo Social sanitario". Revista Trabajo Social y Salud $n^{\circ} 26$.

HEAP, K. (1986): La pratica del lavoro sociale con i gruppi. Un aprrocio sistematico. Astrolabio, Roma.

KIM BERG, I.; MILLER, S. (1996): Trabajando con el problema del alcohol. Gedisa, Barcelona.

KONOPKA, G. (1969): Trabajo Social de Grupo. Euramérica, Madrid.

LAING, R.; PHILLIPSON, H.; RUSSELL LEE, A. (1988): Percepción interpersonal. Amorrortu, Buenos Aires.

MADANES, C. (1997): Violencia Masculina. Granica, Buenos Aires. 
NEIMEYER, R.; MAHONEY, M. (1998): Constructivismo en Psicoterapia. Paidós, Barcelona.

NEUBURGER, R. (1997): La familia dolorosa. Herder, Barcelona.

O HANLON, W.; WEINER-DAVIS, M. (1989): En busca de soluciones. Paidós, Barcelona.

ROSSELL, T. (1997): "Trabajo Social de Grupo: grupos socioterapéuticos y grupos socioeducativos", en Cuadernos de Trabajo Social n' 11 , Universidad Complutense de Madrid, Madrid.

SCHWARTZ,W. (1971): " On the Use of Grups in Social Work Practice", en SCHWARTZ, W. yZALBA, S. (1971): The Practice of Group Work. Columbia University Press, pp. 3-24.

SELEKMAN, M. (1996): Abrir caminos para el cambio, Gedisa, Barcelona.

SHAW, M. (1980): Dinámica de Grupo. Herder, Barcelona.

SLAIKEU,K. (1988): Intervención en crisis. Manual Moderno, México.

STOCKTON, R.; HULSE, D. (1980): Devoluping cohesion in small groups: theory and research. Journal for Specialists in Group Work. $N^{\circ} 6$. USA.

TEVAN, R. (1998): "Grupos de padres de una unidad de cuidados intensivos" Revista Trabajo Social y Salud $n^{\circ} 30$.

TRISELIOTIS, J. (1994): El Trabajo de Grupo en la adopción y acogimiento familiar. Ministerio de Asuntos Sociales, Madrid.

WATZLAWICK; WEAKLAND; FISCH(1982): La táctica del cambio. Herder, Barcelona.

VINTER, R.(1969): La práctica del Servicio Social de Grupo. Humanitas, Buenos Aires.

VV.AA. (1999): Los grupos de autoayuda con personas VIH positivas. Memoria de actividades de la Asociación de Lucha AntiSida de Mallorca, 1999. Alas, Palma.

YALOM, I.(2000): Psicoterapia existencial y Terapia de Grupo. Paidós, Barcelona.

YALOM, I.; VINOGRADOV, S. (1996): Guía breve de psicoterapia de grupo. Paidós, Barcelona. 